according to the 'I NEED HELP' criteria are outlined in table 1. Notably, $69 \%$ of the cohort had $\geq 3$ markers of advanced HF. At the time of clinic review, $28 \%(n=9)$ required urgent admission for further assessment and management, almost all $(89 \%, 25 \%$ of total cohort) of these requiring inotropes. Out of the total cohort, $31 \%$ subsequently underwent MCS implantation (median: $33 \pm 13$ days) or heart transplantation (median $38 \pm 22$ days) (table 2). At time of clinic visit, $37.5 \%$ had a relative contraindication to transplantation with $16 \%$ having $\geq 2$, the most frequently observed being elevated body mass index (BMI) $>30 \mathrm{~kg} / \mathrm{m}^{2}$, excess alcohol intake and renal dysfunction with an estimated glomerular filtration rate $<30 \mathrm{ml} / \mathrm{min} / 1.73 \mathrm{~m}^{2}$.

Conclusion Referrals to the National Advanced HF service in Ireland are presenting at an already markedly advanced stage, with over two-thirds having 3 or more established markers of advanced HF, a quarter requiring direct admission leading to inotropes, and almost a third proceeding to advanced surgical therapies. In addition, more than one third of patients referred had a relative contraindication identified at first consultation, with lifestyle factors accounting for a significant proportion. These findings have implications for development of formal referral pathways and greatly increased resource provision to a hitherto understudied cohort to enable optimal assessment and management for this patient group.

\section{A REVIEW OF PATIENTS WITH HEART FAILURE WITH MID-RANGE EJECTION FRACTION (HFMREF): CO- MORBIDITIES AND OUTCOMES}

${ }^{1} \mathrm{D}$ O' Callaghan, ${ }^{1} \mathrm{P}$ Wheen, ${ }^{2} \mathrm{P}$ Murray, ${ }^{3} \mathrm{C}$ Minelli, ${ }^{1} \mathrm{C}$ Daly. ' St. James's Hospital, Dublin, Ireland; ${ }^{2}$ Tallaght University Hospital, Tallaght, Dublin, Ireland; ${ }^{3} \mathrm{MRHT}$

\subsection{6/heartjn|-2020-ICS.47}

Background The ESC now recognises HF patients with a left ventricular ejection fraction (LVEF) of $40 \%-49 \%$ as having a distinct phenotype, referred to as Heart Failure with midrange Ejection Fraction (HFmrEF).

This classification was implemented to promote research in this group of heart failure (HF) patients.

Aims We aimed to compare the co-morbidities of HFmrEF patients with those of Heart Failure with preserved Ejection Fraction (HFpEF) and Heart Failure with reduced Ejection Fraction (HFrEF) patients, and aimed to compare hospitalisations for acute decompensation HF, and mortality between these groups.

Methods All new referrals to a HF clinic in three neighbouring hospitals were included between January 1st 2017, and December 31st 2017. Data was collected on co-morbidities associated with each HF classification. Mean follow up was 17.5 months $( \pm$ 7.6) and we recorded hospitalisations for $\mathrm{ADHF}$ and mortality data.

Results 286 new patients were referred to one of our $3 \mathrm{HF}$ clinics; following optimisation, the patients were divided as: HFpEF 67 (23.4\%), HFmrEF 58 (20.3\%), HFrEF 94 (32.9\%). 69 patients did not have a repeat echocardiogram. Table 1 presents the co-morbidities. Categorical data was analysed with Chi-square testing and continuous data analysed with unpaired $t$ testing. Significance in HFrEF and HFpEF is in comparison with HFmrEF. Figure 1 is a Kaplan Meier curve demonstrating the events across the follow-up period.

\begin{tabular}{llllll} 
Abstract 47 Table 1 & Co-morbidities & & & \\
\hline & HFmrEF & HFrEF & P value & HFpEF & P value \\
\hline Age, mean (SD) & $69.4(14.6)$ & $66.2(12.1)$ & 0.15 & $72(14.0)$ & 0.39 \\
Ischaemic, n (\%) & $22(39.2)$ & $49(52.1)$ & 0.13 & $28(41.8)$ & 0.78 \\
NYHA I, n (\%) & $14(25)$ & $19(20.2)$ & 0.49 & $16(23.9)$ & 0.89 \\
Diabetes, n (\%) & $13(23.2)$ & $19(20.2)$ & 0.66 & $22(32.8)$ & 0.24 \\
Hypertension, n (\%) & $25(44.6)$ & $49(52.1)$ & 0.38 & $42(62.7)$ & 0.04 \\
MI, n (\%) & $23(41.1)$ & $45(47.9)$ & 0.42 & $19(28.4)$ & 0.14 \\
CABG, n (\%) & $3(5.4)$ & $21(22.3)$ & 0.006 & $12(17.1)$ & 0.03 \\
PCI, n (\%) & $19(33.9)$ & $36(38.3)$ & 0.59 & $17(25.4)$ & 0.30 \\
AFIb/Flutter, n (\%) & $26(46.4)$ & $42(44.7)$ & 0.84 & $38(56.7)$ & 0.26 \\
Hospitalisations (mean (SD)) & $0.23(0.54)$ & $0.34(0.74)$ & 0.37 & $0.34(0.69)$ & 0.35 \\
Mortality, n (\%) & $6(10.7)$ & $12(12.8)$ & 0.71 & $9(13.4)$ & 0.65 \\
\hline
\end{tabular}

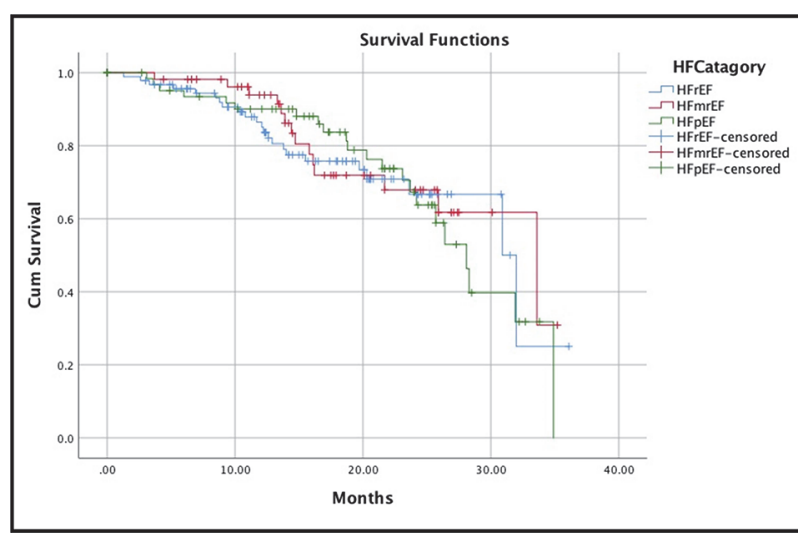

Abstract 47 Figure 1 Events across the follow-up period

Conclusion Co-morbidities were similar in HFmrEF as compared with $\mathrm{HFpEF}$ and $\mathrm{HFrEF}$, aside from previous CABG surgery (fewer in HFmrEF as compared with HFpEF and HFrEF) and HTN (there were more in HFpEF). Mortality was similar across the 3 groups over our follow up period of 17.5 months, and there was no difference in mean hospitalisations for ADHF between the groups. In our catchment area, our HFmrEF have a similar phenotype to our HFpEF and HFrEF cohorts.

\section{TREATMENT OF HEART FAILURE IN 2020: CHAMPS OR CHUMPS?}

R Cusack, A Radhakrishna, J Barton. University Hospital Galway, Galway, Ireland

\subsection{6/heartjnl-2020-ICS.48}

Introduction The importance of achieving target doses of guideline directed medical therapy (GDMT) for patients with heart failure with reduced ejection fraction (HFrEF) is well established. Recently, large registry studies conducted in both the United States (CHAMP-HF) and in Europe (CHECK-HF) have identified while much progress has been made compared with similar studies in the early part of the millennium, significant deficiencies in prescribing trends and adherence to guidelines still remain. Our goal was to conduct an audit of prescribing trends in a tertiary referral centre at University Hospital Galway, within a specialist-led heart failure outpatient 
clinic service, to evaluate our performance in comparison to these large studies.

Methods A retrospective audit was conducted on all patients with HFrEF attending the outpatient heart failure clinic at University Hospital Galway between January and March 2020. Demographic and clinical data included age, gender, ejection fraction, NYHA class, comorbidities (in particular type 2 diabetes), aetiology of cardiomyopathy, history of admissions with decompensation within 12 months, history of ICD/CRT implantation, systolic blood pressure and pulse rate. Biochemical data included estimated glomerular filtration rate, NTproBNP and potassium levels. Medication data included types and doses of GDMT, reasons (if documented) for not being on target dose, loop diuretic doses, and whether the patient was prescribed a SGLT2 inhibitor.

Results Data was collected on a total of 129 patients with a mean age of 71.3 . 69\% were male with a mean LVEF of $28.5 \%$. Overall usage of RAS inhibitors and beta blockers was better than or comparable to existing large scale studies. However, a low proportion of patients on optimal dosing of these therapies was observed, consistent with existing data. A much larger proportion (45.7\%) of our cohort were prescribed an ARNI than in either CHAMP-HF or CHECK-HF, likely a reflection of the approval of the drug occurring partway though the studies. Regrettably our centre performed relatively poorly in both prescription and uptitration of MRAs. In terms of reasons underlying non-prescription or suboptimal dosing, with the exception of ACEI/ARBs no clearly documented or identifiable reason made up the largest proportion of each drug group. Of note only 6 patients $(4.7 \%)$ were prescribed SGLT2 inhibitors; all of these patients were diabetic.

Conclusions Despite the strong body of evidence underpinning GDMT, gaps in prescribing habits still exist with specialist heart failure outpatient services. In particular issues around more widespread use of MRAs continue to undermine optimal therapy for heart failure. The low usage of SGLT2 inhibitors despite compelling evidence from the DAPA-HF trial is likely secondary to the lack of established guidelines on its use; we would expect this to be addressed in the next update to the ESC Guidelines.

\section{ELIGIBILITY FOR DAPAGLIFLOZIN IN A REAL-LIFE HEART FAILURE CLINIC}

C Powell, K Kavanagh, J Morgan, N Stakelum Byrne, P Keelan, N Murphy. Our Lady of Lourdes Hospital, Drogheda, Co. Louth, Ireland

\subsection{6/heartjin-2020-ICS.49}

Introduction The rising prevalence of heart failure due to an aging population, higher post-myocardial infarction survival and the increasing burden of cardio-metabolic diseases, presents public health and economic challenges. Optimisation of heart failure therapy is essential in preventing the morbidity and mortality associated with this disease. The landmark DAPA-HF trial identified a $26 \%$ relative reduction in a composite primary outcome of worsening heart failure or death with use of dapagliflozin in patients with heart failure and reduced ejection fraction (HFrEF), irrespective of diabetic status. Although this class of drug is not yet incorporated into ESC guidelines, such a marked benefit may prompt consideration. The objective of this study is to determine the proportion of patients eligible for dapagliflozin at our site, based on inclusion and exclusion criteria of the DAPA-HF trial.

Methods This retrospective observational study was conducted at Our Lady of Lourdes Hospital, Heart Failure Clinic. Data was collected on all patients referred from January 2018 to December 2019. We employed four data sources: CELLMA, a programme used to record clinical information; PACS (radiology); WinPath (blood work); and patient charts. This multipronged approach served to limit missing data. Eligibility required: $\mathrm{EF} \leq 40 \%$; NYHA $\geq \mathrm{II}$; absence of type 1 diabetes mellitus (T1DM); SBP $\geq 95 \mathrm{mmHg}$; eGFR $\geq 30 \mathrm{ml} / \mathrm{min}$; commensurate elevated BNP levels; and taking at least an ACE inhibitor, $\mathrm{ARB}$ or sacubitril/valsartan combined with a beta blocker (BB). Complete case analysis was used, as data was complete for $>90 \%$ patients.

Results In all, there were 587 referrals during this period, of which 278 (47.4\%) had HFrEF and 130 (22.1\%) had an EF $<40 \%$ (DAPA-HF inclusion). 31\% were female with a mean age of $69 \pm 12$. Of the subset of interest (EF $\leq 40 \%$ ), comorbid T2DM was identified in 38 (29.2\%) and AF in $62(47.7 \%)$. Mean EF was $30.4 \pm 7 \%$ and median BNP was $419 \mathrm{pg} / \mathrm{ml} .102(78.5 \%)$ were receiving at least minimum pharmacological therapy as stated above. 12 (9.2\%) were prescribed fully titrated dosages of ACE inhibitor, BB and mineralocorticoid receptor antagonists. The DAPA-HF inclusion criteria were not met in 68/130 (52.3\%). Of these, 25 were not on minimum pharmacological therapy, 24 were below the BNP threshold, 11 were NYHA I, 10 had an eGFR <30, 14 were hypotensive and 2 had T1DM. Exclusion criteria overlapped in 17 cases. In total, 62 patients were eligible for dapagliflozin, equating to $10.6 \%$ of the full 587 sample, rising to $22.3 \%$ when we focus on the 278 HFrEF patients.

Conclusion In a real-life Heart Failure Clinic almost one quarter of HFrEF patients fulfilled the DAPA-HF inclusion criteria. These patients may stand to benefit from dapagliflozin in terms of reduced hospitalisations and mortality, were this drug prescribed in addition to guideline-recommended heart failure therapies.

\section{CLINICAL INDICATIONS FOR TRANS-THORACIC ECHOCARDIOGRAPHY}

F O'Riordan, C Hart, AM O'Flynn, Anne Hickey. Department of Cardiology, HSE Mallow General Hospital, Co. Cork, HSE, Ireland

\subsection{6/heartjnl-2020-ICS.50}

Background Trans-thoracic echocardiography (TTE) is a commonly performed non-invasive investigation for the assessment of ventricular function and cardiac morphology. The British Society of Echocardiography have established guidance outlining twelve clinical indications in which echocardiography may provide incremental value to patient management (BSE Guidance).

Aims The aim of this study was to audit TTE requests received by the Cardiology Department and compare them against BSE Guidance. Our overall aim is to promote appropriate resource utilisation.

Methods We retrospectively collected all TTE requests received by the Cardiology Department over a four week period from August 2019 to September 2019. We examined the clinical 